\title{
La Organización Mundial de Comercio. Entre las normas del siglo XX y la política comercial del siglo XXI
}

\author{
Julieta Zelicovich*
}

\begin{abstract}
RESUMEN
Las relaciones comerciales internacionales atraviesan un período de transformaciones. A partir del análisis de estadísticas y documentos, se identifica en este trabajo una tendencia hacia la configuración de una nueva política comercial externa a nivel global, con mayores niveles de proteccionismo y uso distorsivo de subsidios. Esta nueva configuración ha provocado renovadas tensiones en las relaciones entre los Estados, planteando el interrogante respecto del tipo de acciones estratégicas que requiere su abordaje. Se argumenta, que el multilateralismo sigue presentado las mayores fortalezas frente a este tipo de problemas. De allí que exista un rol para la Organización Mundial de Comercio, llenando el gap que existe entre las «normas del comercio internacional del siglo XX» y las «nuevas políticas comerciales del siglo XXI».
\end{abstract}

Palabras clave: multilateralismo, política comercial, subsidios, OMC, comercio internacional.

\section{The World Trade Organization: between the XXth century rules and XXIst century Trade Policy}

\section{Abstract}

International trade relations are going through a period of transformation. From the analysis of statistics and documents, a trend towards the configuration of a new foreign trade policy

\footnotetext{
* Licenciada en Relaciones Internacionales por la Universidad Nacional de Rosario, magíster en Relaciones Comerciales Internacionales por la Universidad Nacional de Tres de Febrero y doctora en Relaciones Internacionales por la Universidad Nacional de Rosario. Investigadora en Consejo Nacional de Investigaciones Científicas y Técnicas (CONICET). Profesora de Economía Internacional en la Licenciatura en Relaciones Internacionales de la Universidad Nacional de Rosario. Profesora invitada en la Maestría en Política y Economía Internacionales de la Universidad de San Andrés y en la Maestría en Relaciones Internacionales de la Universidad Nacional de La Plata. Correo electrónico: julieta.zelicovich@fcpolit.unr.edu.ar
}

(iD) https://orcid.org/ 0000-0002-8524-9002 
at a global level, with higher levels of protectionism and distortive use of subsidies, is identified in this work. This new configuration has caused renewed tensions in relations between States, raising the question regarding the type of strategic actions that its approach requires. It is argued that multilateralism continues to present the greatest strengths in the face of this type of problem. Hence, there is a role for the World Trade Organization, filling the gap that exists between the "international trade rules of the $20^{\text {th }}$ century" and the "new trade policies of the $21^{\text {st }}$ century".

Keywords: multilateralism, trade policy, subsidies, WTO, international trade.

\section{Introducción}

A comienzos de 2011 Richard Baldwin publicó la obra $21^{\text {st }}$ Century Regionalism: Filling the gap between $21^{\text {st }}$ century trade and $20^{\text {th }}$ century trade rules. El argumento central es la existencia de una brecha entre el comercio de bienes y servicios del siglo XXI —el cual está estructurado en torno a cadenas globales de valor - y las normas «del siglo XX» — que consagró la Organización Mundial de Comercio (OMC) al finalizar la Ronda Uruguay_- Esa brecha, sostiene Baldwin, ha sido suplida por acuerdos preferenciales profundos, acuerdos bilaterales de inversión y reformas unilaterales, antes que por el foro multilateral. Estos acuerdos se distinguen de otros por haber puesto el foco en el nexo entre «comercio-inversiones-servicios» propio del nuevo siglo, incorporando en los tratados compromisos en - al menos- cuatro áreas: política de competencia, movimientos de capitales, propiedad intelectual e inversiones (Baldwin, 2011). En ese marco analítico se sostiene que el espacio restante para la OMC es limitado: señala Baldwin que los acuerdos profundos ${ }^{1}$ son una amenaza para la OMC porque erosionan la capacidad de esta organización para ser un foro para las negociaciones entre sus miembros, y restan relevancia a su función como mecanismo de solución de controversias. La consecuencia final de este proceso es — conforme a la argumentación del citado autor — una regulación global del comercio más fragmentada, con menor legitimidad, y menor capacidad de obligatoriedad (Baldwin, 2011).

El transcurso de la década parece demostrar que la tesis de Baldwin resultaría correcta: como «respuesta regulatoria a un imperativo tecnoproductivo» (Bouzas, 2017, p. 68), los acuerdos comerciales preferenciales se han incrementado, y en particular lo han hecho los que incluyen compromisos profundos que van más allá de

\footnotetext{
1 En la literatura se refiere a los acuerdos profundos a partir de dos interpretaciones: por un lado, a raíz de la incorporación de regulaciones «más allá de la frontera» — tales como los servicios, las inversiones, la contratación pública, los derechos de propiedad intelectual u obstáculos técnicos—; y por otro, como aquellos que avanzan compromisos más profundos a los de la $\mathrm{OMC}$ - conocidos como OMC+ y OMCx-.
} 
la regulación arancelaria en frontera y comienzan a incorporar obligaciones relativas a ese "comercio del siglo XXI». Por otro lado, si bien en la Conferencia Ministerial de Bali de 2013 los países miembros de la OMC acordaron un Acuerdo sobre Facilitación de Comercio y en la de Nairobi de 2015 avanzaron sobre el Acuerdo de Subvenciones a las Exportaciones Agrícolas, los progresos en una regulación multilateral del comercio han sido más bien escasos y poco ambiciosos.

Ello no ha significado la desaparición de la arena multilateral. La OMC, si bien debilitada, se ha mantenido a flote e incluso, en los últimos ańos, ha comenzado a esbozarse una nueva agenda de discusión en las negociaciones internacionales vinculada a las iniciativas conjuntas de la agenda de Buenos Aires (Hannah, Scott y Wilkinson, 2018). Comercio electrónico, regulación doméstica de servicios, facilitación de inversiones, comercio y género son temas que comienzan a permear la agenda multilateral, insinuando que la regulación por vía de los acuerdos comerciales regionales puede ser insuficiente, o menos adecuada que la multilateral.

A la vez que se avanzaron negociaciones en esta vía, un segundo fenómeno, de características opuestas al anterior ha ido asentándose: en los últimos tres ańos se han incrementado las medidas de proteccionismo comercial; las cuales se han vuelto, además, más complejas. Ello, por su parte, ha incrementado los niveles de conflictividad en las relaciones comerciales internacionales, volviendo a dotar de relevancia y significado a la capacidad de la OMC de administrar los acuerdos negociados y de ser foro para la solución de controversias, postulando nuevos desafíos sobre ello.

Frente a estas dos tendencias, una que muestra el incremento de prácticas proteccionistas y otra que señala cierta vigencia de la OMC como foro para canalizar la cooperación en materia de relaciones comerciales internacionales, este trabajo tiene como meta esbozar el siguiente argumento. Parafraseando a Baldwin, se sostiene que el punto actual de la OMC no pasa por «llenar la brecha entre el comercio del siglo XXI y las normas del siglo XX», cosa que ya han hecho los acuerdos regionales con mejor desempeño que la OMC; sino llenar el gap que existe entre esas «normas del siglo XX» y las «nuevas políticas comerciales del siglo XXI»; variable sobre la que los acuerdos comerciales regionales no llegarían a tener una influencia suficiente, y que se manifiesta como el principal elemento de tensión en las relaciones comerciales internacionales contemporáneas.

Para abordar esta hipótesis el presente trabajo repasa, en primer lugar, los fundamentos de la cooperación multilateral en materia de políticas comerciales; seguidamente, analiza las tendencias de la política comercial a nivel global; en tercer lugar, identifica de qué manera son percibidas estas prácticas como elemento de tensión y problema en las relaciones comerciales internacionales. Por último, explora las 
fortalezas y debilidades del multilateralismo como estrategia para lidiar con la situación contemporánea de las relaciones comerciales internacionales.

El trabajo combina la revisión bibliográfica, el análisis de estadísticas —vinculadas a la aplicación de medidas comerciales-, y el análisis de discursos y documentos, tanto de política comercial de países seleccionados como las actas de los distintos comités de la OMC donde se plantean las preocupaciones comerciales entre los países miembros, y los informes relativos al pedido de consultas en el órgano de solución de controversias.

La investigación se enmarca dentro de un conjunto de trabajos académicos que indagan sobre la reforma de la Organización Mundial de Comercio y también el incremento del proteccionismo en la política comercial externa, tales como los de Bown y Hillman (2019), Hoekman (2019) y Goldstein y van Lieshout (2020), entre otros.

\section{El multilateralismo como piedra angular del sistema multilateral de comercio}

En la historia del sistema multilateral de comercio la inclusión de nuevas temáticas en las negociaciones comerciales internacional ha sido una consecuencia de la evolución en materia de políticas por parte de los países miembros — quienes fueron complejizando los instrumentos de la política comercial—; y la sensibilidad que estas nuevas prácticas en la política comercial provocaban en los mercados - con especial énfasis en EE. UU.- . Por ejemplo, en el trabajo de Zeiler (2012) se da cuenta de esta correlación en la expansión de los contenidos de la regulación de la política comercial externa en momentos específicos de la historia como la Ronda Kennedy, la Ronda Tokio o inclusive la Ronda Uruguay. En cada uno de esos momentos «las partes contratantes reconocieron que el GATT necesitaba cambiar con los tiempos de nuevas demandas, nuevas limitaciones y los cambios en la distribución de la riqueza entre las naciones, incluidos los líderes en el comercio mundial» (p. 369). En este sentido, el GATT mostró durante su historia una gran capacidad de adaptación a los cambios tecnológicos, ideológicos y políticos de cada etapa: primero ante la creación de la Comunidad Económica Europea, luego en las décadas de 1960 y 1970 con la adopción de nuevos proteccionismos asociados a políticas de industrialización vía instrumentos no arancelarios; y en los ochenta con la inclusión de una nueva etapa de expansión del ethos liberal2 ${ }^{2}$ Según Van Grasstek (2013, p. X):

[...] el principal motivo para la transición del GATT a la OMC ha sido precisamente la ampliación de lo que suponemos que es el objeto de la política comercial, ya que

\footnotetext{
2 Véase por ejemplo Gilpin (2001), capítulo 8 «The trading system».
} 
se consideró que el primer acuerdo, que era más un contrato que una institución, era un vehículo insuficiente para dar cabida a las nuevas cuestiones. Sin embargo, la creación de este nuevo vehículo no puso fin a las discusiones sobre lo que constituye el comercio exterior y la política comercial ya que los Miembros de la OMC siguen debatiendo sobre si el sistema podría ampliarse para abarcar nuevas cuestiones, y de qué forma.

En esta evolución, un elemento que se mantuvo constante fue el multilateralismo. En materia de comercio internacional los países tienen incentivos para cooperar (Steinberg, 2008); y durante el período de mayor expansión del comercio mundial esa cooperación fue además multilateral. Conforme a Baldwin, al ser el comercio internacional crecientemente interdependiente y global, la armonización de la regulación del comercio necesaria para su funcionamiento solo puede alcanzarse de manera multilateral (Baldwin, 2016a).

Ruggie ha desarrollado uno de los trabajos más influyentes en la literatura de las Relaciones Internacionales respecto del multilateralismo. Para Ruggie (1992, p. 571), el multilateralismo refiere a un modo de cooperación entre tres o más Estados cuya singularidad radica en estar basado en una serie de principios "generalizados» - generalized — de conductas que suponen una indivisibilidad entre los países que lo integran y cierta cuota de "reciprocidad difusa» ${ }^{3}$, tales como el principio de nación más favorecida, que rige en la OMC.

La convergencia en torno a estos valores, así como la conformación de estrategias nacionales consistentes con ello han sido en la teoría de Ruggie las explicaciones para la creación y sostenimiento del multilateralismo en la segunda parte del siglo XX. La elección de esta estrategia también depende en parte de la configuración de poder internacional, de los intereses en determinada área de cuestiones - la cooperación multilateral es temáticamente específica-, de las preferencias nacionales, y de las estrategias de los demás actores respecto del multilateralismo o bilateralismo (Doran, 2010; Martin, 1993).

El multilateralismo como estrategia para las relaciones internacionales de un Estado presenta una serie de ventajas: es considerado la vía más robusta en términos de «legitimidad», frente al accionar unilateral o bilateral. Además, puede reducir costos de interacción, evitando la reiteración de negociaciones - y concesiones diversas- que supondría una red de acuerdos bilaterales (Zartman y Touval, 2010). La combinación de ambos factores deriva en que «el multilateralismo asegura que varios otros

\footnotetext{
3 Conforme la define Keohane (1986) la reciprocidad difusa, opuesta a la reciprocidad específica, orienta a la conducta del Estado no en función de una recompensa inmediata de actores específicos, sino en razón de la obtención de resultados globales satisfactorios para todo el grupo del que forma parte, como un todo. Se trata de que exista una "equivalencia aproximada de los beneficios en el agregado y en el tiempo" (Ruggie, 1992, p. 571).
} 
Estados aceptarán el liderazgo y las orientaciones de política exterior que adopta el Estado grande» (Doran, 2010, p. 43).

Para los países pequeños el multilateralismo presenta una oportunidad de participación en los procesos de toma de decisión. No obstante, ello también supone que el país o países líderes deberán negociar u ofrecer incentivos para mantener la decisión, lo que en última instancia lleva a incorporar una noción de poder en el análisis de la cooperación multilateral. Así resulta oportuno señalar que «el multilateralismo, aunque es bueno en abstracto para los Estados pequeños y grandes, en la práctica implica compensaciones, que ninguno de los dos puede estar dispuesto a aceptar» (Doran, 2010, p. 43). Asimismo, el multilateralismo queda expuesto a las lógicas de la acción colectiva, tanto en términos de sus beneficios en torno a la maximización de bienes públicos, como de los costos asociados a la defección y el free-rider (Olson, 1965).

El final del siglo XX y las primeras décadas del XXI han traído a la arena de la política internacional un «multilateralismo con adjetivos» que vuelve al escenario más complejo, en tanto nuevas demandas son incorporadas para definir una cierta calidad o performance de dicho multilateralismo. «Los conceptos de equidad, empoderamiento, representación justa e incluso democracia ahora infunden el concepto de multilateralismo» (Osler Hampson, 2010, p. 61). Incluso, como sostiene este último autor, el multilateralismo puede significar cosas distintas para los europeos, los estadounidenses o los latinoamericanos. También encontramos ejemplos de distintos significados de multilateralismo a lo largo de historia del propio sistema multilateral de comercio, donde por ejemplo en realidad la historia del GATT había estado plagada de situaciones de «minilateralismo ${ }^{4} »$ (Kahler, 1992). Dependiendo de los adjetivos que primen, el multilateralismo puede ser más fácil o difícil de alcanzar y mantener.

Como alternativa al multilateralismo, los acuerdos preferenciales aparecen como un escenario subóptimo para muchos temas, incluido el comercial. Como hemos señalado los acuerdos de libre comercio han crecido de manera exponencial en los últimos treinta años, y en parte lo han hecho simplemente porque la vía multilateral resultaba muy compleja o incluso — dada la parálisis de la Ronda Doha— no estaba disponible. Los acuerdos preferenciales pueden resultar consistentes con las normas del sistema multilateral, a través del artículo XXIV del GATT, aunque no lo son con el espíritu de la lógica del GATT-OMC (Goldstein y van Lieshout, 2020). Estos acuerdos, si bien logran mayores niveles de efectividad, así como de profundidad en

\footnotetext{
4 Alude a situaciones de colaboración/cooperación entre un conjunto delimitado de Estados poderosos, dentro de las instituciones bilaterales. El minilateralismo reduce las ganancias de la cooperación, pero incrementa la robustez y posibilidad de la misma. Durante el período del GATT, las potencias del Atlántico habrían disfrazado su minilateralismo de multilateralismo a través del andamiaje institucional (Kahler, 1992).
} 
la complejidad de los temas abordados, tienen sus propias limitaciones: se sostiene que los foros preferenciales carecen de legitimidad multilateral; generan una regulación asimétrica del comercio global, al crear núcleos o nodos de regulaciones normativas lo que encarece los costos para la operación comercial (Pauwelyn y Alschner, 2014; Rosales, Herreros, Frohamann y García-Millán, 2013). Por otro lado, estos acuerdos plurilaterales erosionan la relevancia del mecanismo de solución de diferencias de la OMC (Baldwin, 2016a). En tercer lugar, como señala Hoekman, los acuerdos preferenciales conducen a la consolidación de un conjunto de temas de baja cooperación: normas sobre las que no hay consenso ni en la esfera multilateral ni en la plurilateral, y que quedarían virtualmente excluidas de cualquier esfuerzo de regulación (Hoekman, 2014 en Zelicovich, 2019).

Estas limitaciones han llevado a que, en muchos casos, se buscara la multilateralización de compromisos preferenciales ${ }^{5}$, generando presión sobre la agenda post Doha, además de incorporar nuevos temas al escenario multilateral. El caso del comercio digital es el más reciente y evidente, en ese sentido. En estas negociaciones, se ha propuesto utilizar como base para algunas de las cuestiones negociadas lo acordado en distintos tratados comerciales previos, siguiendo el patrón de acuerdos como los promovidos por ASEAN, o los promovidos por EE. UU. (Janow y Mavroidis, 2019).

\section{Las nuevas políticas comerciales del siglo XXI y la crisis de globalización}

La crisis del orden liberal internacional, que se manifestó en torno a sucesos como la votación por el Brexit en Reino Unido y la política de American First en los EE. UU., ha tenido a la política comercial en el foco de las transformaciones. La política comercial externa se ha convertido en un tema de agenda electoral (Van Grasstek, 2017); convive con otros objetivos y actores de políticas públicas - como, por ejemplo, los ambientalistas- (Osakwe, 2011); y hasta en algunos casos se ha cargado de una «retórica bélica» que deriva en nociones como la de "guerra comercial» en la gestión de la política comercial externa (Harding y Hading, 2020) ${ }^{6}$.

Así como en la década de 1970 emergió un «nuevo proteccionismo» al que este orden liberal internacional buscó responder mediante nuevos acuerdos comerciales internacionales, en los albores de la tercera década del siglo XXI parece emerger una «nueva

\footnotetext{
5 Morin, Brandi y Berger (2019) presentan cinco tipos ideales de multilateralización de acuerdos preferenciales: i) un acuerdo multilateral que reúne las cláusulas más frecuentes utilizadas en los acuerdos preferenciales; ii) apoyo a determinadas cláusulas a partir de su adopción por varios países; iii) la popularización de ciertas cláusulas genera un momento proclive al multilateralismo; iv) el acuerdo multilateral se realiza por la imposición de las preferencias de los países más poderosos; y v) un acuerdo en particular es considerado el más apropiado y se multilateraliza.

6 Este enfoque se traslada también a inversiones. Sumadas a las tendencias de mayor restricción sobre el comercio, Canadá y EE. UU. han incorporado el control y monitoreo sobre la IED, con fundamentos en la seguridad nacional.
} 
política comercial». Esta nueva política comercial, como signo de época, implica la apelación mucho más intensa y compleja de medidas de subsidios y prácticas de vinculación entre Estado y mercado que entran en zonas grises de la legislación actual y que resultan problemáticas para las relaciones comerciales internacionales. A ello se le suma, además, la aplicación de medidas proteccionistas «tradicionales» que ya cuentan con un denso marco normativo en la OMC como suba de aranceles, restricciones cuantitativas y licencias. Para Evenett y Fritz (2019) se trata de una política comercial de la era del populismo. Sin apelar a ese término, también Harding y Hading (2020) sugieren que se trata de una nueva etapa singular en la historia de la globalización.

Existen tres rasgos sistémicos a resaltar del período bajo análisis que afectarán el estudio realizado en esta sección: la concentración, la dinámica, y la composición. Dada la distribución - concentrada - del comercio mundial, las políticas de los países que integran el listado de los diez principales exportadores e importadores del mundo, tienen un efecto sustantivo sobre los flujos del comercio mundial -el 53,3\% en 2018-. Así cobran especial atención para el análisis las medidas de EE. UU., China y la Unión Europea; y también Japón, Corea, México, Canadá, Singapur e India.

En segundo lugar, señalamos que en la última década se ha experimentado un período de crecimiento más lento del comercio mundial. En promedio, desde la Segunda Guerra Mundial el comercio tuvo un crecimiento anual de 1,5 veces el PBI global. Desde 1990 a 2008 la elasticidad fue mayor y alcanzó a crecer al doble que el PBI mundial. Desde 2011 a 2016 se redujo a 1, y en 2016 fue de 0,8, revirtiendo la pendiente anteriormente observada. Por su parte, el comercio de servicios tuvo un incremento sostenido pasando del 7\% del PBI global en 1991 a 12,6\% en 2016.

El tercer punto alude a la composición de esos flujos comerciales. En ese período una transformación significativa ha sido la de China, que ha pasado a ocupar el primer lugar en el ranking y a exportar productos de mayor valor ańadido (OMC, 2019). Ello, sumado a las transformaciones de tipo tecnológicas (Baldwin, 2016b; Baldwin, 2019), ha generado presiones sobre la política comercial.

En ese sistema de comercio internacional, el rasgo más sobresaliente es la apelación a un mayor proteccionismo en la política comercial externa. Conforme muestran los estudios de monitoreo, entre enero de 2017 y diciembre de 2019 se implementaron a nivel mundial 2723 medidas distorsivas sobre el comercio (Global Trade Alert, 27 de marzo de 2019). Estas medidas tenían protagonismo en la relación entre EE. UU. y China, pero no fueron exclusivamente estos dos actores los que aplicaron un mayor

\footnotetext{
7 En términos del documento estratégico de política comercial emitido por el propio gobierno chino, se trata de pasar de una economía de rápido crecimiento a uno de alta calidad de desarrollo.
} 
nivel de proteccionismo, sino que se trató de un fenómeno más extendido alcanzando al menos a los primeros diez exportadores e importadores a nivel global (Evenett y Fritz, 2019). Para la OMC, a octubre de 2019 el 7,5\% de las importaciones mundiales estaban afectadas por medidas que restringen las importaciones, en un valor estimado de USD 1700 miles de millones (OMC, 2019). Una década atrás la cobertura de este tipo de medidas era de tan solo $0,6 \%$. Se trata de una tendencia ascendente que se acentúa sobre el final de la década (gráfico 1).

Gráfico 1. Cobertura de las restricciones al comercio

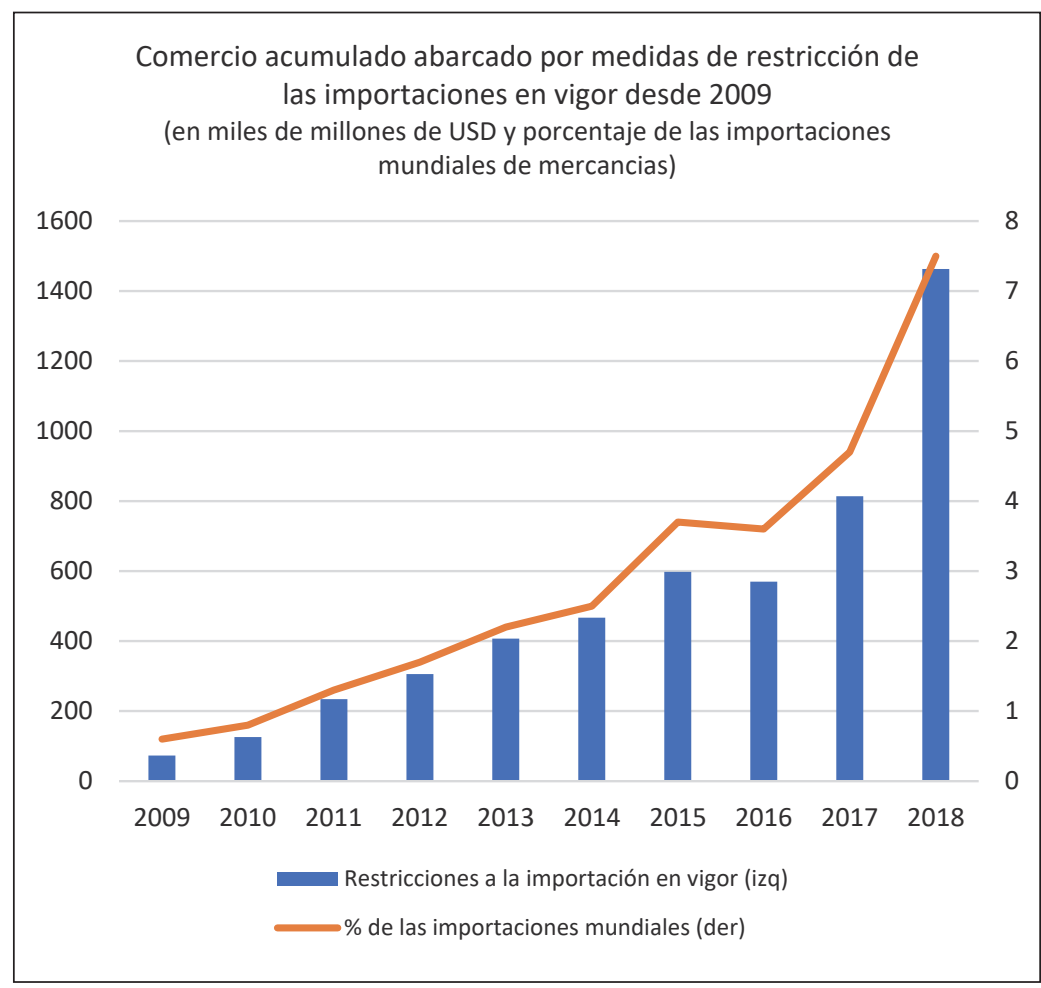

Fuente: Secretaría de la OMC.

Como señalábamos anteriormente, una parte importante de estas medidas son "medidas en frontera», es decir, que afectan a los bienes y servicios en el momento de ingresar al territorio aduanero. Según la OMC, las 102 medidas restrictivas del comercio — con efecto sobre 747000 millones de USD_ aplicadas entre octubre de 2018 y octubre de 2019, fueron en su mayoría aranceles y restricciones cuantitativas a las importaciones (gráfico 2). Los aranceles tuvieron la particularidad de ser fuertemente discriminatorios, teniendo países específicos como objetivo de ese incre- 
mento arancelario. Por su parte, el volumen de esas iniciativas muchas veces superó los USD 10000 millones, llevando a ser catalogado como "proteccionismo Jumbo" (Evenett y Fritz, 2019). Ambos aspectos: direccionalidad y volumen, diferencia al uso de aranceles de esta etapa de las precedentes.

Gráfico 2. Medidas restrictivas del comercio, por tipo

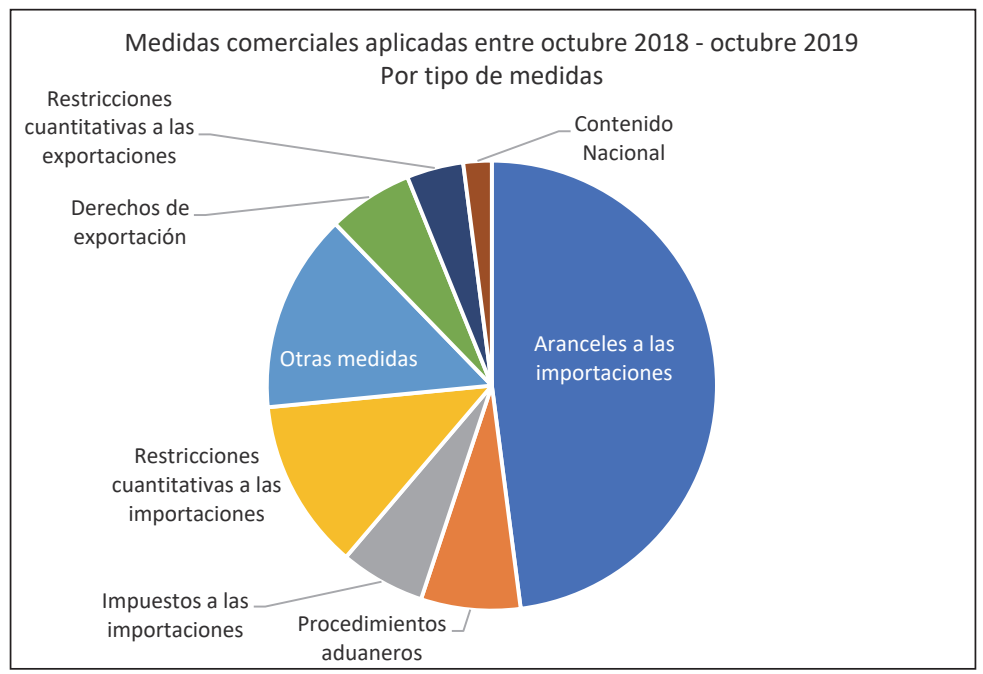

Fuente: OMC (2019).

Las medidas antidumping se han mantenido dentro de sus valores promedio. Las medidas de derechos compensatorios, en cambio, se incrementaron en 2017 y 2018 respectivamente, llegando a duplicar la cantidad de investigaciones iniciadas en 2012, por ejemplo. En esta tendencia, los metales fueron el sector económico que más atención suscitó, vinculado a la sobreproducción de aluminio y acero que antecedió a la guerra comercial. De hecho, EE. UU. es el responsable de más del 50\% de las investigaciones iniciadas en materia de derechos compensatorios, habiendo sido esta (la del uso de subsidios y de derechos compensatorios como respuesta) una de las cuestiones pilares — y más disputada — de la nueva era de la política comercial.

Los subsidios, limitados por la OMC tanto en el acuerdo del GATT como en el Acuerdo de Subvenciones y Medidas Compensatorias, han tenido un desarrollo creciente el último tiempo. Para algunos autores, esta mayor relevancia se debe al rol creciente de China en la producción y el comercio mundial (Bown y Hillman, 2019; Wu, 2016; Chiang, 2018). Este mecanismo, permite a los productores locales mantenerse competitivos frente a las importaciones, y eventualmente, convertirse en competidores globales en sí mismos. 
Según las notificaciones ${ }^{8}$ ante la Organización Mundial de Comercio, en el último año, [...] entre los sectores que recibieron desembolsos importantes durante el período objeto de examen figuran la ayuda a los agricultores, la inversión en programas tecnológicos y de innovación, y la inversión en diversos sectores energéticos. Cabe mencionar también los planes de ayuda al transporte, las garantías de préstamos para las pymes (a menudo vinculadas a la exportación), los mecanismos de ayuda a los proveedores de servicios de telecomunicaciones, las políticas fiscales favorables y diversas políticas de ayuda al sector siderúrgico. (OMC, 2019, p. 91)

Para el CEPR, entre 2017 y finales de 2019, un cuarto del comercio mundial estaba afectado por iniciativas de promoción de exportaciones subsidiadas por los gobiernos (Evenett y Fritz, 2019). El registro de Global Trade Alert muestra que desde 2005 a 2020 se identificaron en el mundo 4105 medidas de subsidios — sin contar subvenciones a las exportaciones- y 1552 medidas relativas a la promoción de exportaciones —incluyendo aquí subsidios—. En total, ambas categorías implican el 64,8\% de los instrumentos políticos considerados por este observatorio como "dañinos» (Global Trade Alert, 27 de marzo de 2019) —aunque conforme los datos indicados supra no todas estas medidas son notificadas o relevadas por la OMC- (gráfico 3).

\section{Gráfico 3. Uso de subsidios en la política comercial}

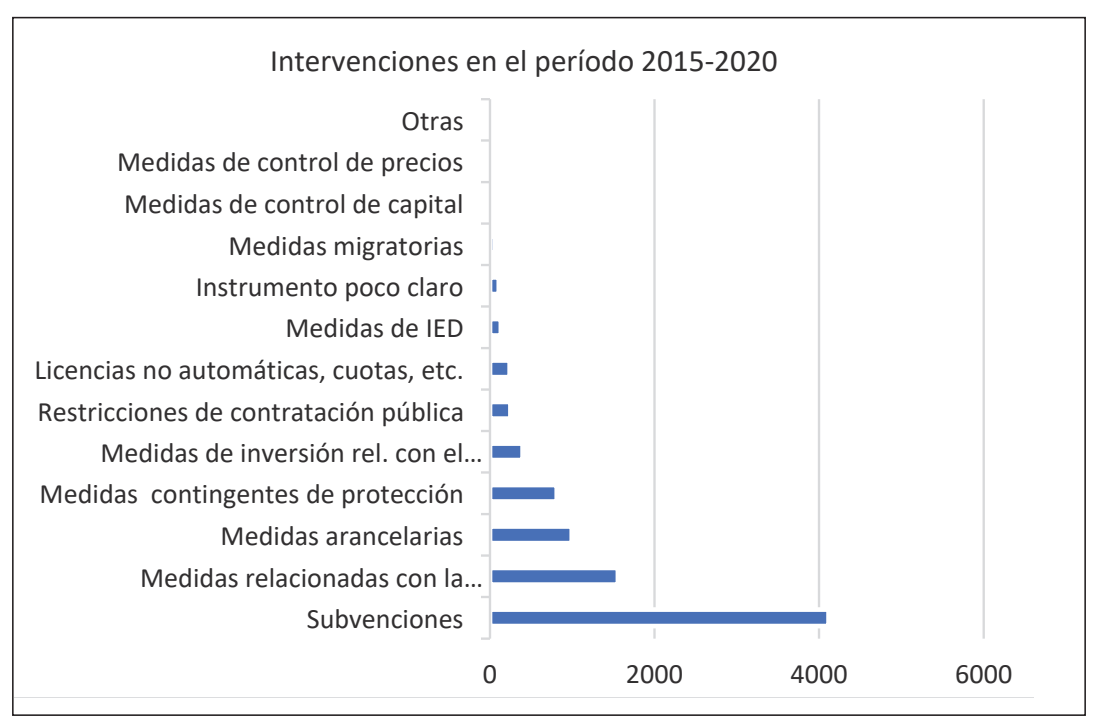

Fuente: Global Trade Alert, 27 de marzo de 2019.

\footnotetext{
8 Un punto de alta sensibilidad es que esta información resulta solo parcial, puesto que se registra un elevado incumplimiento en las notificaciones asociadas a subsidios y subvenciones en la OMC.
} 
Más allá de las diferencias de medición, la tendencia parece poder aprehenderse claramente: el uso de subsidios y prácticas de apoyo similares se ha incrementado y alcanza volúmenes significativos del comercio mundial. Entre los más resonantes encontramos que, por ejemplo, EE. UU. puso en vigencia un paquete de ayuda de USD 12000 millones para los productores agrícolas perjudicados por los trastornos del mercado y los aranceles de represalia en el marco de la guerra comercial con EE. UU. También existe preocupación en torno al programa Made in China 2025, según el cual, a través de un fuerte apoyo estatal, China se propone modificar su rol en la economía mundial. "Los objetivos de la estrategia en tres fases son que China llegue a ser un importante país manufacturero para 2025 (fase 1); que pueda competir con las potencias manufactureras desarrolladas para 2035 (fase 2); y que se convierta en una potencia manufacturera de primer orden para 2049 (fase 3)» (OMC, 2018, p. 158). Para ello, se estima que los subsidios directos a la producción rondaran los cientos de billones de dólares —además de otras estrategias como empresas estatales, y transferencia de tecnología - (McBride y Chatzky, 2019).

El mapeo de los rasgos de las tendencias de la política comercial a nivel global queda incompleto si no se incorporan las medidas destinadas a facilitar el comercio, sea por la aplicación del Acuerdo de Facilitación del Comercio, como por reformas unilaterales emprendidas por el Estado. Entre las medidas relevadas se identifica la implementación de ventanilla única de comercio exterior, así como la remoción de licencias y aranceles, en algunos casos. Conforme a la OMC:

El valor del comercio abarcado por las medidas de facilitación de las importaciones aplicadas durante el período examinado se ha estimado en USD 544.700 millones [...]. Las nuevas medidas de facilitación de las importaciones se orientaron principalmente a los siguientes sectores: máquinas, aparatos y artefactos mecánicos (Capítulo 84 del SA) 13,4\%; máquinas, aparatos y material eléctrico (Capítulo 85 del SA) 12,1\%; cobre y sus manufacturas (Capítulo 74 del SA) 7,6\%; y vehículos automóviles (Capítulo 87 del SA) 7,4\%. (OMC, 2019, pp. 8-9)

Esta tendencia hacia la facilitación se acompaña con la expansión de acuerdos de libre comercio y asociaciones estratégicas. En los últimos cinco años se notificaron a la OMC 52 nuevos acuerdos preferenciales - sobre un total acumulado de 301 desde 1995- La tendencia en esa dirección es acentuada, dando muestras de una preferencia por el bilateralismo (Harding y Hading, 2020), consistente con la erosión de la reciprocidad difusa y las orientaciones de política exterior de tipo «regionalismo estratégico con agenda del siglo XXI».

Se trata de procesos negociadores que se distinguen por cuatro características específicas: i) reúnen países con una participación sustantiva en el comercio mundial; ii) presentan contenidos referidos como OMC+ y OMCx, con especial énfasis en la agenda de lo que Baldwin denomina «regionalismo del siglo XXI» (Política de 
competencia; Movimientos de capital; Propiedad intelectual; Inversiones); iii) tienen como meta generar patrones regulatorios que se extiendan luego a otros, especialmente en el ámbito de la OMC, respondiendo a una estrategia de poder internacional en la disputa por la gobernanza global del comercio internacional; y iv) se centran fundamentalmente en las redes globales de producción. (Zelicovich, 2018, p. 703)

La Unión Europea, Japón y China son los que más activamente han desarrollado esta estrategia en los últimos años. Así acuerdos como el de Unión Europea-Canadá, Unión Europea-Japón, el Tratado Integral y Progresista de Asociación Transpacífico (CPTPP), o el Acuerdo Regional de Asociación Económica Global (RCEP) van en esta línea. Sobre fines de 2019 se agregan a los acuerdos preferenciales un nuevo tipo: los miniacuerdos comerciales promovidos por la administración Trump, a partir del acuerdo entre EE. UU. y Japón.

\section{Elementos de tensión en las relaciones comerciales internacionales}

Algunos de los rasgos de esta nueva política comercial externa que asoma al concluir la segunda década del siglo XXI han sido más problemáticos que otros. A partir del análisis de los documentos de política comercial de las principales economías y de las actas de los comités de la OMC, así como de las consultas iniciadas en el órgano de solución de controversias este apartado presenta un perfil de aquello que genera disrupciones en las relaciones comerciales internacionales. El solo hecho de que durante los primeros nueve meses de 2019, los países solicitaran la celebración de consultas en un número de casos equivalente a los registrados en la totalidad de 2016 y 2017 (OMC, 2019) da cuenta de este mayor nivel de tensión en el ámbito del comercio global —además de dar cuenta de la vigencia, a pesar de las crisis, del foro de la OMC como mecanismo para solución de controversias-.

De la lectura de esos documentos se desprende que, en el último año, las regulaciones sobre la prohibición o restricción de determinados productos tecnológicos (5G), la restricción a exportaciones entre ciertos mercados (EE. UU.-China), incluidos los aranceles, y las regulaciones sobre propiedad intelectual y denominaciones de origen ocuparon la mayor parte de las cuestiones planteadas por los miembros en la OMC. En segundo término, fue recurrente la mención en las actas a las cuestiones vinculadas a medidas antidumping. Por último, constituye un elemento constante - puesto que se hace alusión a reclamos anteriores - la realización de observaciones relativas a los instrumentos distorsivos en las políticas agrícolas, tanto en ayuda interna como en subvenciones a las exportaciones.

El incremento de aranceles y restricciones cuantitativas han sido una de las medidas que, naturalmente, introdujeron tensión en las relaciones comerciales internacionales. 
En este caso, la dinámica ha sido más focalizada y bilateral —aunque con reverberaciones en la Organización Mundial de Comercio-. Las sucesivas subas de aranceles entre EE. UU. y China - y las medidas adicionales impuestas por EE. UU. a otros países en el marco de su fair trade policy— han sido las que más reclamos han suscitado. Se identifican dos tipos de respuestas: las político-diplomáticas y las jurídicas. En términos diplomáticos, se destaca el documento China's Position on the China-US Economic and Trade Consultations del gobierno de China, de julio de 2019. En términos jurídicos, en el marco de la OMC Corea presentó una demanda por las salvaguardas en lavarropas y paneles de energía solar; México, Canadá, India, Unión Europea y China, solicitaron, cada uno de forma separada, el establecimiento de un grupo especial para dirimir acerca de las medidas implementadas al aluminio y al acero. Posteriormente, China agregó dos demandas por medidas arancelarias de EE. UU. y EE. UU. inició una demanda contra India, también por aranceles.

En el marco del sistema multilateral de comercio, otro núcleo de preocupación reiterado ha sido el incumplimiento con las medidas de notificaciones. Esto se ha debido en parte, a falta de capacidad especialmente entre los países menos desarrollados, pero en otra parte a voluntad de los países. Según los registros «entre 1995 y 2019 el porcentaje de Miembros que notificaron subvenciones se mantuvo entre el $37 \%$ y el 50\%» (OMC, 2019). Ello ha llevado a EE. UU. y otros a postular ante el Consejo General, una propuesta sobre transparencia ${ }^{9}$. La misma refuerza los mecanismos de creación de capacidades, la posibilidad de contranotificación y la inclusión de una tipología de sanciones ante incumplimiento ${ }^{10}$.

Detrás de la inquietud por las notificaciones se encuentra el elemento que mayores tensiones provoca en las relaciones comerciales internacionales contemporáneas. Se trata de la utilización de los subsidios, y de su regulación en el marco de la OMC. Acorde a una buena parte de la literatura específica la definición de subsidio comprendida en los textos del GATT y la OMC no resulta lo suficientemente precisa, y no cubre el uso de los actuales subsidios — ahora problemáticos—.

Para Bown y Hillman (2019) el problema detrás de los subsidios es tanto su definición, como la carga de la prueba en la aplicación de un subsidio o su medida compensatoria, así como la ineficiencia de estas últimas para contrarrestar los efectos del primero $^{11}$. El subsidio en la OMC es definido inicialmente en el GATT como

\footnotetext{
9 Se trata del documento JOB/GC/204, del 1 de noviembre de 2018.

${ }^{10}$ En cambio, el Grupo Africano, Bolivia, Cuba, Ecuador, India y Omán, se han manifestado en contra de ampliar las obligaciones de notificación, y han enfatizado los problemas en materia de capacidad para cumplimiento de esta obligación. (OMC - Consejo General, 2019).

11 Según estos autores, el efecto de las medidas compensatorias como mecanismo para contrarrestar los subsidios es limitado, puesto que dichas medidas solo pueden ser colocadas si existe dentro del país importador una producción
} 
«una forma de sostenimiento de los ingresos o de los precios que tiene como fin el aumento de las exportaciones o la reducción de las importaciones». Posteriormente, se sostuvo que se trata de «una contribución financiera de un gobierno o de cualquier organismo público en el territorio de un Miembro» que implique una transferencia de fondos como préstamos o garantías, una condonación al pago de ingresos públicos como las bonificaciones fiscales, la proporción de bienes o servicios - sin incluir la infraestructura- o el pago a través de mecanismos de financiación. Estas actividades las puede desarrollar un gobierno, o entidad pública, o bien un privado a pedido de este $(\mathrm{OMC}, 1995)^{12}$. Las empresas estatales integran un universo complejo de clasificar dentro de esas definiciones, especialmente al considerar el caso chino (Wu, 2016). Por su parte, pueden referirse «políticas gubernamentales que crean el efecto de un subsidio, como la aplicación diferencial de los impuestos a la exportación y la devolución diferencial de los impuestos al valor agregado para los insumos y productos en la cadena de suministro de una industria, que no se ajustan a la definición legal actual de un subsidio» (Bown y Hillman, 2019, p. 2).

De hecho, uno de los casos más resonantes de la historia del órgano de solución de controversias involucra la política de subsidios: el caso de subsidios para grandes aeronaves civiles que se suscitó entre EE. UU. y la UE, que finalmente autorizó a los EE. UU. a aplicar contramedidas por USD 7496623 millones anuales, a fines de $2019^{13}$. Otros casos vinculados a subsidios se han centrado en el sector agrícola, como por ejemplo «DS511: China - Ayuda interna para los productores agropecuarios» donde se constató que China hacía un uso excesivo de las medidas de subsidio, por sobre el nivel de «de minimis» ${ }^{14}$. En total, desde la creación de la OMC existen 130 casos en los que se cita el acuerdo sobre subvenciones y medidas compensatorias — sobre un total de 573 casos-.

Dentro de la vía diplomática, este ha sido uno de los temas centrales — junto a transferencia forzada de tecnología — abordados por las reuniones «trilaterales» que

\footnotetext{
de un bien equivalente al subsidiado por el país extranjero, y si logra demostrarse que dicha importación subsidiada genera dańo a la industria.

${ }_{12}$ Un punto importante es el rol del órgano de solución de controversias. Según el gobierno de los EE. UU. "El Órgano de Apelación interpretó el término "organismo público" de una manera indebidamente estrecha que amenazaba la capacidad de las economías de mercado de contrarrestar los subsidios que distorsionan el comercio proporcionados por economías no de mercado" (USTR, 2020).

${ }^{13}$ DS316: Comunidades Europeas y determinados Estados miembros - Medidas que afectan al comercio de grandes aeronaves civiles. Disponible en https://www.wto.org/spanish/tratop_s/dispu_s/cases_s/ds316_s.htm

${ }^{14}$ Conforme lo define el Acuerdo sobre Agricultura, «de minimis» alude a cantidades mínimas de ayuda interna causante de distorsión del comercio que todos los Miembros de la OMC pueden conceder, calculadas como porcentaje del valor de producción (hasta el 5\% para los países desarrollados miembros y hasta el 10\% para los países en desarrollo miembros). Hay niveles de minimis para productos específicos y niveles de minimis no referidos a productos específicos.
} 
EE. UU., Japón y la Unión Europea vienen realizando desde 2017. A lo largo de dichos encuentros se ha ido delimitando una agenda de trabajo compartida entre estos tres actores. La misma alude a proponer la restricción de nuevos subsidios específicos en una eventual reforma del Acuerdo de Subvenciones y Medidas Compensatorias, que incluya especialmente a las "garantías ilimitadas, el otorgamiento de subsidios a empresas insolventes, la asignación de subsidios a empresas que operan en sectores con exceso de capacidad y que no pueden obtener financiamiento de fuentes comerciales independientes, y el perdón directo de cuotas de deuda» (Joint Statement of the Trilateral Meeting of the Trade Ministers of Japan, the United States and the European Union, 14 de enero de 2020). Asimismo se propone en estos documentos mejorar el sistema de notificaciones - algo que se relaciona con el punto anterior- $y$ revertir la carga de la prueba, de manera que sea quien subsidia el que deba demostrar que su política no genera efectos distorsivos en los mercados. Estos aspectos han sido a su vez incluidos - y en términos semejantes - en el documento de lineamientos para la modernización de la OMC elaborado por la Comisión Europea en 2018, y que han orientado su accionar en la esfera multilateral desde entonces.

Si bien, como mencionamos, el programa chino de Made in China 2025 y los problemas asociados con la sobrecapacidad en las industrias de acero y aluminio son la principal preocupación de la "trilateral», China no es el único caso de subsidios. El reporte de barreras al comercio y la inversión de la Unión Europea, incluye también la US Tax Cuts and Jobs Act 2017 como caso con subsidios prohibidos (Comisión Europea, 2019a). Frente a ello, una de las respuestas del bloque europeo frente al crecimiento de este tipo de medidas ha sido la reforma de su procedimiento para medidas antidumping y medidas compensatorias, buscando volver el mecanismo más ágil, y lograr imponer niveles de contramedidas más elevadas ${ }^{15}$.

No puede dejar de señalarse que este debate está superpuesto a otro: el de si pueden en la OMC convivir distintos modelos de desarrollo o debe converger todo hacia un único modelo como "campo de juego nivelado", popularizado en inglés como level playing field. A mediados de 2018 se produjo un intercambio de documentos entre EE. UU. y China en el marco de la OMC en torno a esta cuestión ${ }^{16}$. Para EE. UU. «en la actualidad China mantiene un modelo económico dirigido por el Estado que causa perturbaciones del comercio, no se basa en esos principios fundamentales e impone costos sustanciales a los miembros de la OMC, a los que plantea graves problemas». China, frente a ello, sostuvo que "China respeta y defiende firmemente

\footnotetext{
${ }^{15}$ Un resumen de las notificaciones puede consultarse en https://trade.ec.europa.eu/doclib/docs/2018/june/ tradoc_156921.pdf

${ }^{16}$ La comunicación de los EE. UU. es la WT/GC/W/745, del 11 de julio de 2018. La respuesta de China es la comunicación WT/GC/W/749, del 18 de julio de 2018.
} 
las normas de la OMC, y respalda un sistema multilateral de comercio abierto, transparente, inclusivo y no discriminatorio. China ha participado en todos los aspectos de la labor de la OMC, ha hecho escuchar su voz y ha formulado sus propias propuestas sobre la mejora de la gobernanza económica mundial». Posteriormente, en febrero de 2020, EE. UU. presentó otro documento señalando «la importancia de las condiciones orientadas al mercado para el sistema multilateral de comercio» (WWT/GC/W/796). Asimismo, la disputa tuvo una vía jurídica también, en tanto que China, en 2016 había presentado la solicitud para establecer una controversia tanto con EE. UU. como con la UE en torno a la determinación del valor normal y el reconocimiento de economía de mercado/no economía de mercado. Al poner el modelo de desarrollo chino en el centro de la disputa, y el tipo de relaciones que otros Estados mantienen con este, el caso suscitó mucha atención. Sin embargo, y en el marco de los diálogos con EE. UU. y la UE, en junio de 2019 China solicitó suspender el caso.

Un segundo desprendimiento de este debate, en tensión en las relaciones comerciales internacionales actuales, es si los países pueden continuar o no manteniendo el derecho a auto designarse como países en desarrollo en la OMC. El promotor de este debate es EE. UU., que propone que aquellos países que sean considerados como de ingresos altos en el Banco Mundial, o que tengan un porcentaje mayor al 0,5\% del comercio mundial, sean miembros de la UE, del G20, o de la OECD —o estén aplicando para integrar esta última-, no puedan declararse países en desarrollo. La UE ha postulado enfoques de gradualidad específicos en este debate. En tanto que China lo considera como un aspecto no negociable ${ }^{17}$.

El funcionamiento de la $\mathrm{OMC}$ ha sido un problema en sí mismo para las relaciones comerciales internacionales. Antecedida por la parálisis de la Ronda Doha, la crisis de la $\mathrm{OMC}$ se ha acentuado en los últimos cinco años. El funcionamiento del órgano de apelaciones (y el bloqueo por parte de EE. UU. de dicho órgano) ha sido identificado como un problema, así como la falta de capacidad de respuesta ante las nuevas prácticas de la política comercial. Tanto EE. UU., como la UE y China aluden al funcionamiento de esta organización, como «problema» en sus documentos de política comercial externa. En el caso de la UE, sostiene que la OMC necesita mejorar su capacidad de control en materia de subsidio y de prácticas desleales, que ello implica resolver cuestiones relativas a la falta de transparencia y responder «a los desarrollos tecnológicos de nuestro tiempo» (Comisión Europea, 2019b, p. 8). EE. UU. alude a tres fallas del sistema: la falla de convergencia — referida a los casos que no son considerados economías de mercado—, la falla de desarrollo — aduciendo que la

${ }^{17}$ En esta posición ha presentado un documento junto a India, Cuba y Venezuela. 
OMC tiene estándares que están desactualizados, y con particular énfasis en la revisión del status de país en desarrollo- y una falla de su capacidad de hacer cumplir las normas - relativas a notificaciones, como a incumplimientos del Órgano de Apelaciones- (USTR, 2020). Desde mediados de 2018 la reforma de la OMC ha ganado peso en la agenda multilateral, estando presente en todas las declaraciones de este foro y también de otros, como el del G20. La falta de mecanismos fortalecidos de cooperación multilateral para las relaciones comerciales internacionales resulta así un problema ya identificado e incorporado en la agenda diplomática global.

Por último, y dando cuenta de la complejidad de la política comercial, se identifica como área de problema la política impositiva respecto a los servicios digitales. Sin ser estrictamente una política comercial, estas medidas tienen incidencia sobre el comercio de servicios y sobre los flujos de bienes digitales. Mientras que Francia ha avanzado en una medida de impuestos, y la OECD ha hecho de este uno de sus núcleos de trabajo, EE. UU. identifica a esta política como una medida injusta y discriminatoria (USTR, 2020). Vinculada a esta medida, se desarrollan las negociaciones - plurilaterales - sobre comercio electrónico, en el marco de las Iniciativas Conjuntas de la Organización Mundial de Comercio, en donde además de las cuestiones impositivas los países negociadores han discutido en torno a la inclusión $-\mathrm{O}$ no- de cláusulas relativas a la localización de servidores, infraestructura, y protección contra la piratería, entre otras cosas.

\section{5. ¿Cuál es el lugar del multilateralismo en las relaciones comerciales internacionales?}

En un trabajo del año 2008, Pauwelyn se preguntaba si la OMC seguía siendo necesaria; doce ańos más tarde, la interrogante que emerge más bien es si la OMC sigue siendo posible (Pauwelyn , 2008). A pesar de la crisis que lo atraviesa, el multilateralismo sigue siendo el mejor foro deseable para canalizar la cooperación en estas tensiones que afectan las relaciones comerciales internacionales. En términos de legitimidad y eficiencia, conforme analizamos en el primer apartado, el multilateralismo presenta sustantivas ventajas para lidiar con estos problemas.

Frente al conjunto de prácticas de las políticas comerciales del siglo XXI el multilateralismo se presenta como la mejor opción. Ante el problema de la transparencia el multilateralismo es una respuesta más eficiente que la preferencial. La previsibilidad y certidumbre sobre el comercio mundial se garantiza solo con un sistema amplio y sistemático de notificaciones, algo que resulta mucho más fácil y eficiente de conseguir en el plano multilateral que a través de una red de acuerdos parciales. También en materia de subvenciones, un esquema de regulaciones más claras solo podrá 
funcionar sobre la legitimidad del multilateralismo y escala del multilateralismo, y no así en enfoque bilateral. Por ejemplo, conforme a los datos de la base Design of trade agreements (Dür, Baccini y Elsig, 2014) solo el 38\% de los acuerdos comerciales tienen una referencia a la restricción en el uso de subsidios» y solo el $0,4 \%$ de los casos $^{18}$ contemplan la eliminación de los subsidios ${ }^{19}$.

El abordaje de la certidumbre del comercio global como bien público, y su sustento sobre la reciprocidad difusa, sigue vigente como mejor mecanismo en la teoría para resolver los problemas. Sin embargo, siguiendo la literatura mencionada, lo teóricamente deseable, no siempre resulta posible.

Las tensiones políticas, las diferencias ideológicas en torno a la relación comerciodesarrollo, y la redistribución de poder global —entre otras - obran en contra del funcionamiento de la salida multilateral, al menos en su forma GATT-OMC previa a la crisis de 2008 (Goldstein y van Lieshout, 2020; Zelicovich, 2019). Conforme Van Grasstek «en la etapa del GATT, la Cuadrilateral, y en especial los Estados Unidos, no solo tenían intereses económicos que les motivaban para crear y mantener el sistema sino también tenían la influencia necesaria para lograr ese objetivo» (2013, p. 31). Actualmente ese diseño decisorio no resulta disponible. En el escenario previo a la Conferencia Ministerial de Nur Sultan, planeada para 2020 pero luego suspendida por el brote de COVID-19, el núcleo decisorio esbozaba una constelación donde hay cuatro o cinco vértices -EE. UU., Japón, Unión Europea, China y posiblemente India en algunos temas - pero estos no forman una figura con sinergias como tenía el QUAD. Esto genera un obstáculo para tomar nuevas decisiones. En efecto parecen ser muy endebles los principios generalizables y pautas de conducta en torno a los cuales los gobiernos de estos cuatro/cinco países puedan converger. Por su parte, la creciente tensión en las relaciones comerciales internacionales incrementa el trabajo del órgano de solución de controversias, haciendo que este se vea sobrecargado, lo cual retroalimenta las tensiones ante la falta de respuesta.

Sin embargo, en todos los documentos analizados los países sí mantienen un discurso de compromisos con el multilateralismo. E incluso, algunos valores en torno a cómo se define el multilateralismo. Las diferencias emergen en el nivel de principios para la acción, siendo la de mayor tensión la de la relación Estado-mercado. De allí que puedan identificarse algunos núcleos que den sustento al multilateralismo debilitado sobre el que funciona el sistema multilateral de comercio, hasta que un nuevo momento de impulso pueda ser más claro —en el mismo sentido que marca

\footnotetext{
${ }^{18}$ Los acuerdos Nueva Zelanda-Singapur del año 2000 y Australia-Singapur de 2003.

19 De hecho, tanto los subsidios a la industria, como la ciberseguridad, dos temas claves para los EE. UU. no lograron ser contemplados en el acuerdo bilateral de Primera Fase que este firmó a fines de 2019 con China.
} 
la historia del GATT- El hecho de que las fricciones y controversias sigan anclándose en el órgano de solución de controversias es un hecho positivo, así como que al menos la mitad los países miembros siga cumpliendo con las notificaciones y participe de las negociaciones por nuevos temas. Se trata entonces de un multilateralismo en disputa.

Desde la perspectiva — más pesimista - de Acharya, nos encontramos en un mundo multiplex, donde «el multilateralismo se está convirtiendo en un complejo mosaico de estructuras separadas y superpuestas de gobernanza global, plurilateral, instituciones y redes regionales, iniciativas del sector privado, sociedad civil, movimientos y asociación público-privadas» (Acharya, 2018, p. 158). Se trata de tiempos donde el multilateralismo posible es más endeble, conflictivo y volátil, aunque no menos necesario. La noción de indivisibilidad del régimen de comercio como bien público continúa siendo estructurante de los desafíos en las relaciones comerciales internacionales; en tanto que los principios generalizados de conducta están siendo revisados - ya sea en términos de la reciprocidad difusa, como en cuanto a un nivel más específico de la relación entre política comercial y desarrollo-. La posibilidad del multilateralismo, depende pues de la convergencia de esos principios.

\section{Conclusiones}

A lo largo del trabajo se ha identificado una serie de tendencias que marcan un nuevo momento en las relaciones comerciales internacionales. Estas tendencias aluden a una renovada dimensión en el uso de subsidios y de las barreras arancelarias, pero también al incremento de las medidas correctivas —antidumping y compensatorias - con una perspectiva de proteccionismo. En simultáneo se observa una propensión hacia la incorporación de facilitación de comercio y de negociaciones de acuerdos preferenciales, pero sobre una base bilateral o minilateral. El análisis empírico y teórico muestra que se trata de una configuración de políticas comerciales distinta a la de períodos previos.

En particular la transparencia y el uso de subsidios de nuevo tipo son identificados como elementos de tensión en las relaciones comerciales internacionales. Frente a ellos el multilateralismo aparece como el mejor instrumento para su abordaje por sobre el de los acuerdos comerciales preferenciales-. Esa opción bilateral o preferencial como instrumento de la gobernanza global presenta limitaciones. Los acuerdos comerciales regionales pueden resultar insuficientes para lidiar con parte de las tensiones mencionadas anteriormente (subsidios, aranceles a gran escala, transparencia), y de allí el interés de las principales economías en trasladarlos a la esfera multilateral. El rol del multilateralismo no está en el gap entre «el comercio del 
siglo XXI» y las "normas del siglo XX", sino en la brecha existente entre esas "normas del siglo XX» y las nuevas prácticas de la "política comercial global en el siglo XXI». El multilateralismo teóricamente necesario y relevante, no es sin embargo, aun políticamente posible, dada la distribución de poder mundial y las preferencias nacionales en torno a los liderazgos, especialmente en un contexto donde la política comercial externa se encuentra fuertemente politizada. Frente a ello nos encontramos en un multilateralismo de transición, que debe encontrar los principios sobre los cuales volver a erigir una cooperación internacional más sólida en materia de relaciones comerciales internacionales.

El trabajo aquí realizado contribuye a señalar la vigencia del multilateralismo, a la vez que invitar a reflexionar más sistemáticamente respecto de cuáles serían los incentivos y requisitos necesarios para su funcionamiento, tanto en las relaciones comerciales internacionales como en otras áreas temáticas específicas.

\section{Referencias bibliográficas}

Acharya, A. (2018). Constructing Global Order. Agency and Change in World Politics. Cambridge: Cambridge University Press.

Baldwin, R. (2011). 21t century Regionalism: Filling the gap between $21^{\text {st }}$ century trade and $20^{\text {th }}$ century trade rules. Ginebra: World Trade Organization.

Baldwin, R. (2016a). The World Trade Organization and the future of multilateralism. Journal of Economic Perspectives, 30(1), 95-116.

Baldwin, R. (2016b). The Great Convergence. Information, Technology and the New Globalization. Londres: The Belknap Press of Harvard University Press.

Baldwin, R. (2019). The Globotics Upheaval. Globalization, Robotics and the Future of Work. Londres: Widenfeld \& Nicolson.

Bouzas, R. (2017). El regionalismo en América Latina y el Caribe: ¿qué hay de nuevo? Estudios Internacionales, (49), 65-88.

Bown, C., y Hillman, J. (2019). WTO'ing a Resolution to the China Subsidy Problem. Peterson Institute for International Economics.

Chiang, T.-W. (2018). Chinese state-owned enterprises and WTO's anty-subsidy regime. Goergetown Journal of International Law, 49, 845-886.

Comisión Europea. (2019a). EU Trade Policy at Work. Luxemburgo: Publications Office of the European Union. Recuperado de https://trade.ec.europa.eu/doclib/docs/2019/ october/tradoc_158400.pdf

Comision Europea. (2019b). Report form the Commission to the Parliament and the Council on Trade and Investment Barriers. 1 january 2018-31 December 2018. Bruselas: Comisión Europea. Recuperado de https://trade.ec.europa.eu/doclib/docs/2019/ june/tradoc_157929.pdf 
Doran, C. (2010). The two sides of multilateral cooperation. En W. Zartman y S. Touval, International Cooperation. The Extents and Limits of Multilateralism (pp. 40-59). Cambridge: Cambridge University Press.

Dür, A., Baccini, L. y Elsig, M. (2014). The Design of International Trade Agreements: introducing a new database. Review of International Organizations, 9(3), 353-375.

Evenett, S. y Fritz, J. (2019). Going it Alone? Trade policy after three years of populism. . Londrés: CEPR Press, Max Schmidheny Foundation, Global Trade Alert.

Gilpin, R. (2001). Global Political Economy. Understanding the international economic order. Princeton: Princeton University Press.

Global Trade Alert. (27 de marzo de 2019). Global Dynamics. Recuperado de https://www. globaltradealert.org/global_dynamics/area_all/year-from_2015

Goldstein, J. y van Lieshout, E. (2020). Is there a future for Multilateral Trade Agreements? En A. Klasen. The Handbook of Global Trade Policy. Oxford: John Wiley \& Sons.

Hannah, E., Scott, J. y Wilkinson, R. (2018). The WTO in Buenos Aires: The outcome and its significance for the future of the multilateral trading system. World Economy, 41(10), 1-21. https://doi.org/10.1111/twec.12657

Harding, R. y Hading, J. (2020). Strategic Trade as a Means to Global Influence. En A. Klasen, The Handbook of Global Trade Policy. Oxford : John Wiley \& Sons.

Hoekman, B. (2014). Supply Chains, Megarregionals and Multilateralism: a road map for the WTO. London: CEPR Press.

Hoekman, B. (2019). Urgent and Important: Improving WTO Performance by Revisiting Working Practices. Journal of World Trade, 53(3), 373-394. Recuperado de http:// respect.eui.eu/wp-content/uploads/sites/6/2019/04/Hoekman_WTO_Reform_ JWT_pre-publication-version.pdf

Höppner, M. (2020). The Global Trade Environment - A new reality. En A. Klasen, The Handbook of Global Trade Policy (pp. 25-46). Oxford: John Wiley \& Sons.

Janow, M. y Mavroidis, P. (2019). Digital Trade, E-commerce, the WTO and regional frameworks. World Trade Review, 18(S1), s1-s7. https://doi.org/10.1017/ S1474745618000526

Joint Statement of the Trilateral Meeting of the Trade Ministers of Japan, the United States and the European Union. (14 de enero de 2020). Washington. Recuperado de https:// www.meti.go.jp/press/2019/01/20200115001/20200115001-2.pdf

Kahler, M. (1992). Multilateralism with small and large numbers. En J. Ruggie, Multilateralism Matters. The theory and praxis of an institutional form. Nueva York: Columbia University Press.

Keohane, R. (1986). Reciprocity in International Relations. International Organization, 40(1), 1-27.

Martin, L. (1993). The Rational State Choice of Multilateralism. En J. Ruggie, Multilateralism Matters. The theory and praxis of an institutional form (pp. 91-124). Nueva York: Columbia University Press.

McBride,J.yChatzky,A.(2019,05 13). Is “madeinchina2025” athreattoglobaltrade?Recuperado de https://www.cfr.org/backgrounder/made-china-2025-threat-global-trade 
Morin, J., Brandi, C. y Berger, A. (2019). The Multilateralization of PTAs' Environmental Clauses: Scenarios for the Future? En M. Elsig, M. Hahn y G. Spilker. The Shifting Landscape fo Global Trade Governance: World Trade Forum (pp. 207-232). Cambride: Cambridge University Press.

Olson, M. (1965). The logic of collective action. Public goods and the theory of groups. Cambridge: Harvard University Press.

OMC - Organización Mundial de Comercio. (2019). WT/GC/W/778/Rev.2 - Fortalecer la OMC para promover el desarrollo y la inclusión. Comunicación del Grupo Africano, el Estado Plirnacional de Bolivia, Cuba, el Ecuador, India y Oman. Ginebra: OMC.

OMC - Organización Mundial de Comercio. (1995). Acuerdo sobre Subvenciones y Medidas Compensatorias. Recuperado de https://www.wto.org/spanish/docs_s/legal_s/24scm.pdf

OMC - Organización Mundial de Comercio. (2018). Examen de las políticas comerciales. Informe de la secretaría. China. WT/TPR/S/375. Ginebra: Órgano de Examen de las Políticas Comerciales.

OMC - Organización Mundial de Comercio. (2019a). Informe del Director General al OEPC sobre los hechos ocurridos recientemente en relación con el comercio. De mediados de octubre de de 2018 a medidados de mayo 2019. WT/TPR/OV/W/13. Ginebra: OMC.

OMC - Organización Mundial de Comercio. (2019b). Revista general de la evolución del entorno comerical internacional. Informe anual del Director General. Octubre 2018Octubre 2019. WT/TPR/OV/22. Ginebra: OMC. Recuperado de https://docs.wto. org/dol2fe/Pages/SS/directdoc.aspx?filename=s:/WT/TPR/OV22.pdf

Osakwe, C. (2011). Developing countries and GATT/WTO Rules: Dynamic Transformations in Trade Policy Behavior and Performance. Minnesota Journal of International Law, 20(2), 365-436.

Osler Hampson, F. (2010). Deconstructing multilateral cooperation. En W. Zartman y S. Touval, International Cooperation. The extents and limits of multilateralism (pp. 60-77). Cambridge: Cambridge University Press.

Pauwelyn, J. (2008). New Trade Politics for the 21st century. Journal of International Economic Law, 11(3), 559-573.

Pauwelyn, J. y Alschner, W. (2014). Forget about the WTO: the network of relations between preferential trade agreements and double PTAs. Ginebra: Graduate Institute of International and Development Studies.

Rosales, O., Herreros, S., Frohamann, A. y García-Millán, T. (2013). Las negociaciones megarregionales: hacia una nueva gobernanza del comercio mundial. Santiago de Chile: CEPAL.

Ruggie, J. (1992). Multilateralism: the Anatomy of an Institution. International Organization, 46(3), 561-598.

Steinberg, F. (2008). Cooperación y conflicto en las relaciones económicas internacionales. Revista Española de Ciencia Politica, (18), 149-176.

USTR. (2020). 2020 Trade Policy Agenda and 2019 Anual Report of the President of the United States on the Trade Agreements Program. Washington: Gobierno de Estados Unidos. 
VanGrasstek, C. (2013). Historia y futuro de la Organización Mundial del Comercio. Ginebra: Organización Mundial del Comercio.

VanGrasstek, C. (2017). Back to the Future: US Trade Policy under the Trump Adminsitration. En C. Primo Braga y B. Hoekman, Future of the Global Trade Order (pp. 49-80). San Domenico de Fiesole: EUI, IMD, FDC.

Wu, M. (2016). The "China, Inc." challenge to global trade governance. Harvard International Law Journal, 57(2), 262-324.

Zartman, W. y Touval, S. (2010). Introduction: return to the theories of cooperation. En W. Zartman y T. Saadia, International Cooperation: The extents and limits of multilateralism (pp. 1-15). Cambridge: Cambridge University Press.

Zeiler, T. (2012). The expanding mandate of the GATT: The first seven rounds. En A. Narlikar, M. Daunton y R. Stern. The Oxford Handbook on the World Trade Organization. Oxford: Oxford University Press.

Zelicovich, J. (2018). Usos estratégicos de las negociaciones comerciales intenracionales en un orden de hegemonías en disputa. Brazilian Journal of International Relations, 7(3), 692-717.

Zelicovich, J. (2019). La crisis de la OMC y el trilema de las negociaciones comerciales internacionales. Relaciones Internacionales, 92(1), 1-21. https://doi.org/10.15359/ ri.92-1.1

Recibido: 30 de marzo de 2020

Aprobado: 5 de julio de 2020 